\title{
EVOLUCIÓN TERRITORIAL DE LA TRANSICIÓN EPIDEMIOLÓGICA EN EL ESTADO DE MÉXICO,
} 1980-2015

\section{TERRITORIAL EVOLUTION OF THE EPIDEMIOLOGICAL TRANSITION IN THE STATE OF MEXICO, 1980-2015}

\author{
Jesús Emilio Hernández Bernal \\ Universidad Autónoma del Estado de México \\ jesemili@hotmail.com \\ Marcela Virginia Santana Juárez \\ Universidad Autónoma del Estado de México \\ mvsantana7@hotmail.com \\ Noel Bonfilio Pineda Jaimes \\ Universidad Autónoma del Estado de México \\ noelpj2000@gmail.com \\ Jesús Gastón Gutiérrez Cedillo \\ Universidad Autónoma del Estado de México \\ iggc1321@yahoo.com.mx \\ Diana Gómez Barroso \\ Centro Nacional de Epidemiología. Instituto de Salud Carlos III. CIBERESP \\ dgomez@externos.isciii.es
}

\begin{abstract}
RESUMEN
El propósito de este artículo es analizar la distribución territorial de la transición epidemiológica (únicamente mortalidad) en el Estado de México, durante el período de 1980 a 2015. Esta variable presenta un progreso evolutivo, diferenciado a lo largo del tiempo y del territorio. Los indicadores tomados en cuenta en este estudio son las tasas de mortalidad que se deben a alguna de las enfermedades incluidas en la lista GBD 165 (Global Burden Disease, o Carga Global de Enfermedad) desarrollada por la OMS. Estas tasas se calcularon con base en la población total y las defunciones hospitalarias registradas del periodo 1980-2015. Posteriormente se analizaron estadísticamente empleando como métodos el Valor Índice Medio (VIM) y el análisis bivariado, cuya interpretación permitió determinar el comportamiento espacio temporal de la transición epidemiológica y elaborar la cartografía correspondiente. Algunos hallazgos señalan que en el Estado de México la transición epidemiológica de la mortalidad presenta cuatro etapas: dos de ellas en regiones extensas en el territorio y otras dos en pequeñas regiones, de forma aleatoria.
\end{abstract}

Palabras clave: Transición. Epidemiología. Lista GBD. Bivariado. Mortalidad.

\begin{abstract}
The aim of this article is to analyze the territorial distribution of the epidemiological transition (mortality only) in the State of Mexico, during the period $1980-2015$. This variable shows an evolution and a differentiated progress along time and territory. The indicators used in this study are mortality rate due to diseases belonging to the GBD 165 (Global Burden Disease) list, developed by $\mathrm{WHO}$. These rates were calculated based on the total population and the registered hospital deaths of the period 1980-2015. Later, the data was statistically analyzed
\end{abstract}

Recebido em: 09/12/2020

Aceito para publicação em: 29/04/2021. 
Evolución territorial de la transición epidemiológica en el estado de México, 1980-2015
Jesús Emilio Hernández Bernal

Marcela Virginia Santana Juárez

Noel Bonfilio Pineda Jaimes

Jesús Gastón Gutiérrez Cedillo

Diana Gómez Barroso

using the Mean Index Value (MIV) and the bivariate analysis, whose interpretation allowed us to determine the spatio-temporal behavior of the epidemiological transition and to elaborate the corresponding cartography. Some findings point out that in the State of Mexico, the epidemiological transition of mortality presents four stages: two of them in extensive regions in the territory and another two in small regions in a random way.

Keywords: Transition. Epidemiology. GBD list. Bivariate. Mortality.

\section{INTRODUCCIÓN}

La transición epidemiológica para el Estado de México ha ocurrido de una manera cambiante a lo largo del tiempo, y su comportamiento territorial es desconocido. El objetivo de este trabajo es la determinación del comportamiento de las diversas etapas de la transición epidemiológica (específicamente mortalidad) para el Estado de México, y su distribución territorial.

Como base teórica, se tomó la teoría clásica de la transición epidemiológica. Esta considera los cambios que se presentan en los patrones de salud y su relación con los determinantes sociales, económicos y demográficos, proporcionando una descripción y una explicación a los cambios que se observan en las tasas de mortalidad y morbilidad. En esta investigación se tomó en cuenta únicamente la mortalidad (defunciones hospitalarias), y se analizó su comportamiento evolutivo y cambios registrados a lo largo del tiempo. Los datos empleados fueron obtenidos a partir de fuentes oficiales como el Sistema Nacional de Información en Salud (SINAIS) y el Instituto Nacional de Estadística y Geografía (INEGI). Del SINAIS se retomó la lista de enfermedades GBD 165, de la cual se extrajeron las causas de muerte codificadas. Estas se presentan en cuatro grandes grupos: I. Enfermedades transmisibles, maternas, perinatales y nutricionales; II. Enfermedades no transmisibles; III. Causas externas de morbilidad y mortalidad; y IV. Causas mal definidas. Para este estudio, se tomaron en cuenta los dos primeros grupos de causas, y se obtuvieron sus tasas de mortalidad respectivas durante el período de 1980-2015.

Para analizar el comportamiento de las tasas de mortalidad para cada causa, se utilizaron los métodos del Valor Índice Medio (VIM) y el análisis bivariado. El primero consiste en la estandarización y clasificación de los datos mediante la curva de distribución normal o campana de Gauss y, posteriormente, la obtención del promedio (VIM) de los datos obtenidos. Como se mencionó, se clasificaron las diferentes causas de muerte en dos grupos, de manera que, para la clasificación por periodos, se empleó solo el VIM para cada grupo. En cuanto al segundo método, consiste en analizar las causas de muerte, comparando la distribución de la mortalidad del grupo I con respecto a la del grupo II.

Algunos de los hallazgos sobre la distribución territorial de la transición epidemiológica en el Estado de México, señalan que las etapas de transición epidemiológica se presentan en forma heterogénea en el territorio.

\section{CONSIDERACIONES TEÓRICAS}

Esta investigación se fundamenta en la teórica clásica de la transición epidemiológica propuesta por Omran en 1971. Algunos de los postulados son los siguientes:

La transición epidemiológica se refiere a los cambios a largo plazo en los patrones de muerte, enfermedad e invalidez que caracterizan a una población específica. Por lo general, estos cambios se presentan junto con transformaciones demográficas, sociales y económicas. Esta transición es un concepto dinámico que se centra en la evolución de la mortalidad y morbilidad, e implica un cambio en la ocurrencia de enfermedades infecciosas asociadas con carencias primarias, así como en la aparición de enfermedades crónicas degenerativas, que se relacionan con factores genéticos y con la existencia de carencias secundarias (Frenk et al., 1991). Hoy en día, se analizan los cambios y los riesgos tecnológicos que pueden influir en la transición epidemiológica (Kuri-Morales, 2011). 
Evolución territorial de la transición epidemiológica en el estado de México, 1980-2015
Jesús Emilio Hernández Bernal Marcela Virginia Santana Juárez

Noel Bonfilio Pineda Jaimes Jesús Gastón Gutiérrez Cedillo Diana Gómez Barroso

El concepto de transición epidemiológica fue propuesto por Abdel R. Omran en 1971, para quien la transición epidemiológica corresponde a los cambios que se producen en los patrones de muerte y enfermedad. La teoría clásica de la transición epidemiológica determina la existencia de tres fases, que reciben su nombre a partir de la causa de muerte dominante. Estas fases son: la edad de la peste y el hambre (etapa inicial), la edad de las pandemias retraídas (etapa de transición) y la edad de enfermedades degenerativas y las causadas por el hombre (etapa final) (Vera Bolaños, 2000, figura 1).

Figura 1 - Transición epidemiológica propuesta por Omran, 2020. Elaboración propia con base en Omran, 2020.

\section{Fase 1}

Fase 2

Fase 3

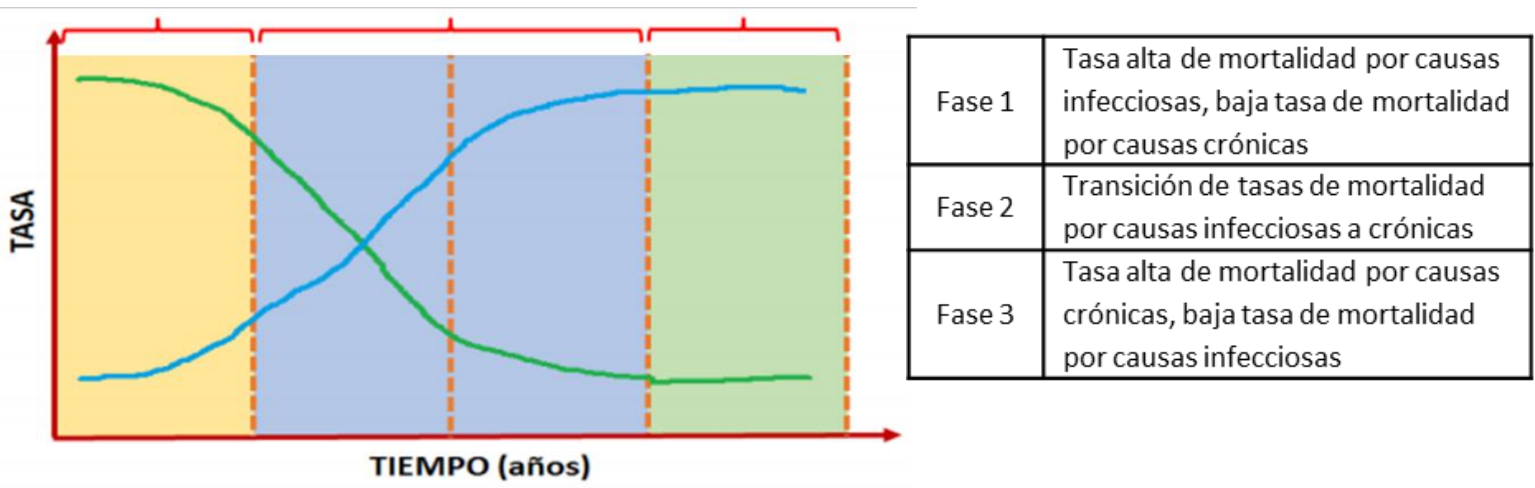

De acuerdo a las fases propuestas por Omran, la fase 1 presenta una tasa de mortalidad elevada, por causa de epidemias y enfermedades infecciosas, mientras que hay tasas muy bajas de mortalidad por enfermedades crónicas. La fase 2 es la etapa de transición, donde las tasas de mortalidad por enfermedades infecciosas disminuyen y las tasas de mortalidad por enfermedades crónicas aumentan. Por último, en la fase 3 los papeles se invierten con respecto a la fase 1: las tasas de mortalidad por enfermedades infecciosas disminuyen a un número muy bajo de defunciones, mientras que las tasas de mortalidad por enfermedades crónicas degenerativas se colocan en los primeros lugares (Vera Bolaños, 2000).

La definición clásica fue modificada por Lerner en 1973, el propuso que la transición epidemiológica fuera presentada como un proceso continuo, en el cual los patrones de salud y enfermedad de una sociedad se van transformando en respuesta de los cambios de carácter demográfico, socioeconómico, tecnológico, político y cultural (Eliecer Andrade, 2011).

Para esta investigación, se elaboró un modelo propio, basado en el modelo clásico propuesto por Omran. Nuestro modelo comienza a partir de una segunda etapa de la transición epidemiológica de la mortalidad, la cual se caracteriza por presentar una alta tasa de mortalidad debida a la causa 1 y una baja tasa de mortalidad debida a la causa 2. Le sigue una tercera etapa, en la que encontramos una baja mortalidad de la causa 1 y una alta mortalidad de la causa 2, y, seguidamente, encontramos la etapa de transición óptima, que presenta una baja mortalidad por ambas causas. Se finaliza con una etapa de transición regresiva, en la cual hay una alta mortalidad por ambas causas (figura 2). 
Evolución territorial de la transición epidemiológica en el estado de México, 1980-2015
Jesús Emilio Hernández Bernal

Marcela Virginia Santana Juárez

Noel Bonfilio Pineda Jaimes

Jesús Gastón Gutiérrez Cedillo

Diana Gómez Barroso

Figura 2 - Transición epidemiológica, 2020. Elaboración propia con base en Omran, Vera y Frenk, 2020.

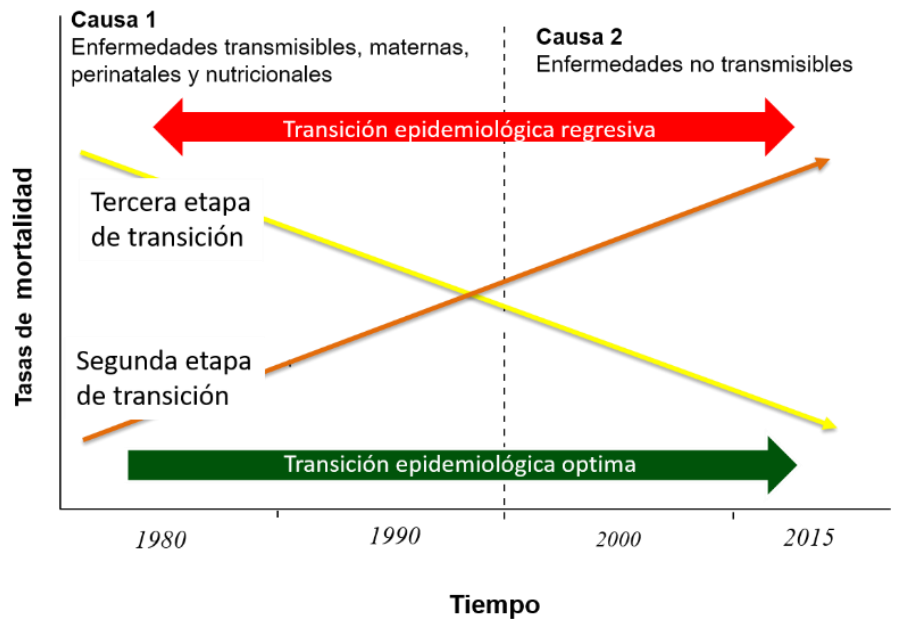

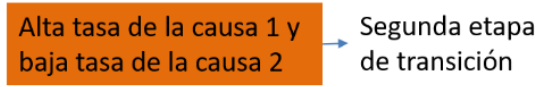

Baja tasa de la causa 1 y Tercera etapa de alta tasa de la causa $2 \rightarrow$ transición
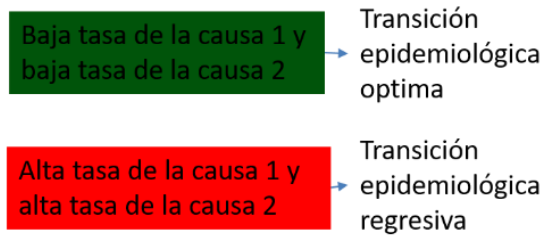

En la investigación denominada: "Análisis comparativo entre la teoría de la transición demográfica y epidemiológica en Argentina”, elaborada por Villavicencio llegó a la conclusión que Argentina ha alcanzado la cuarta fase del modelo de transición demográfica, presentando unas tasas de natalidad y tasas de mortalidad en un proceso de descenso estable. Desde la perspectiva de la transición epidemiológica concluyó que el caso argentino no es un ejemplo del modelo clásico de la transición que se observa en los países europeos, porque Argentina alcanzó la tercera fase del modelo, en donde predominan las enfermedades crónicas y degenerativas, como las cardiovasculares y los tumores, propias de una población envejecida, esta situación no permaneció estable produciéndose un retroceso hacia fases ya superadas del modelo de Omran (Villavicencio, 2006). Este análisis hace la consideración que su transición epidemiológica tiene un retroceso y se asemeja al modelo propuesto por Frenk.

En la investigación "Diferencias geográficas de la mortalidad según grandes causas en la República Argentina (2000-2007) y en la provincia de San Juan (2000-2007)", se analizó la mortalidad en la República Argentina dentro de la primera década del siglo XXI, de acuerdo a la teoría de la transición epidemiológica propuesta en los años setenta por Omran. El objetivo primordial de esta investigación fue el de analizar la mortalidad en la República de Argentina y San Juan según grandes grupos de causas entre los años 2000 y 2007 a fin de establecer patrones espaciales de distribución; de esta investigación se concluyó que Argentina presenta un modelo de transición epidemiológica similar al modelo europeo clásico, está cumpliendo con las cuatro fases en al menos 90 años. Respecto a la Provincia de San Juan, existe un predominio de las enfermedades crónicas y degenerativas, y persiste la mortalidad por infecciosas y parasitarias, además de presentar muertes por desnutrición, (Del Carmen Acosta, 2013). Esta investigación que se retoma se considera por la forma en que se establecieron los patrones espaciales de la distribución epidemiológica y la forma de representación territorial.

En la publicación "Transición demográfica y epidemiológica en el estado de Veracruz, México" se elaboró un análisis cuantitativo y esquemático del proceso de transición demográfica y epidemiológica; también se planteó que la transición demográfica comenzó con la disminución de la mortalidad durante el siglo XIX, y la transición epidemiológica se manifestó a finales del mismo; esta transición epidemiológica se vio marcada por el control de los padecimientos prevenibles por la vacunación y por la erradicación de ciertos vectores, esto permitió un marcado descenso en la disminución de la mortalidad, hasta que surgió la mortalidad por causas de enfermedades infectocontagiosas, la cual fue superada por las patologías crónico-degenerativas. En esta publicación se retomaron fuentes de información como los padrones y registros de población del siglo XIX, los censos de población del Estado de Veracruz, así como las 
estadísticas vitales publicadas por organizaciones oficiales estatales y nacionales del siglo XX (Méndez, 2014). Esta investigación permitió la elaboración de una comparación con los resultados de dos estados pertenecientes a México.

\section{METODOLOGÍA}

Para el análisis de la transición epidemiológica y su distribución territorial en el Estado de México, se emplearon una serie de métodos, que se describen a continuación.

En primer lugar, se elaboró una recopilación de datos de defunciones, junto con las causas de muertes registradas, para el periodo de 1980 a 2015 en el Estado de México. Las causas de muerte se tomaron de la lista de enfermedades GBD 165 (Global Burden Disease, o Carga Global de Enfermedad) desarrollada por la OMS. Asimismo, se recopiló la información de población total en el mismo territorio y periodo de tiempo.

Posteriormente, se procesaron los datos de defunciones y sus respectivas causas de mortalidad, y se obtuvieron las diversas tasas. Se aplicó el método del Valor Índice Medio (VIM) a cada una de las tasas, y al VIM resultante se le aplicó el método de análisis bivariado. El resultado es la generación del mapa de la distribución territorial de la transición epidemiológica, respecto a la causa 1 y la causa 2, para el Estado de México. El proceso se muestra en la figura 3. 
Figura 3 - Esquema metodológico para el análisis de la transición epidemiológica en el Estado de México para el periodo 1980 - 2015. Elaboración propia, 2020.

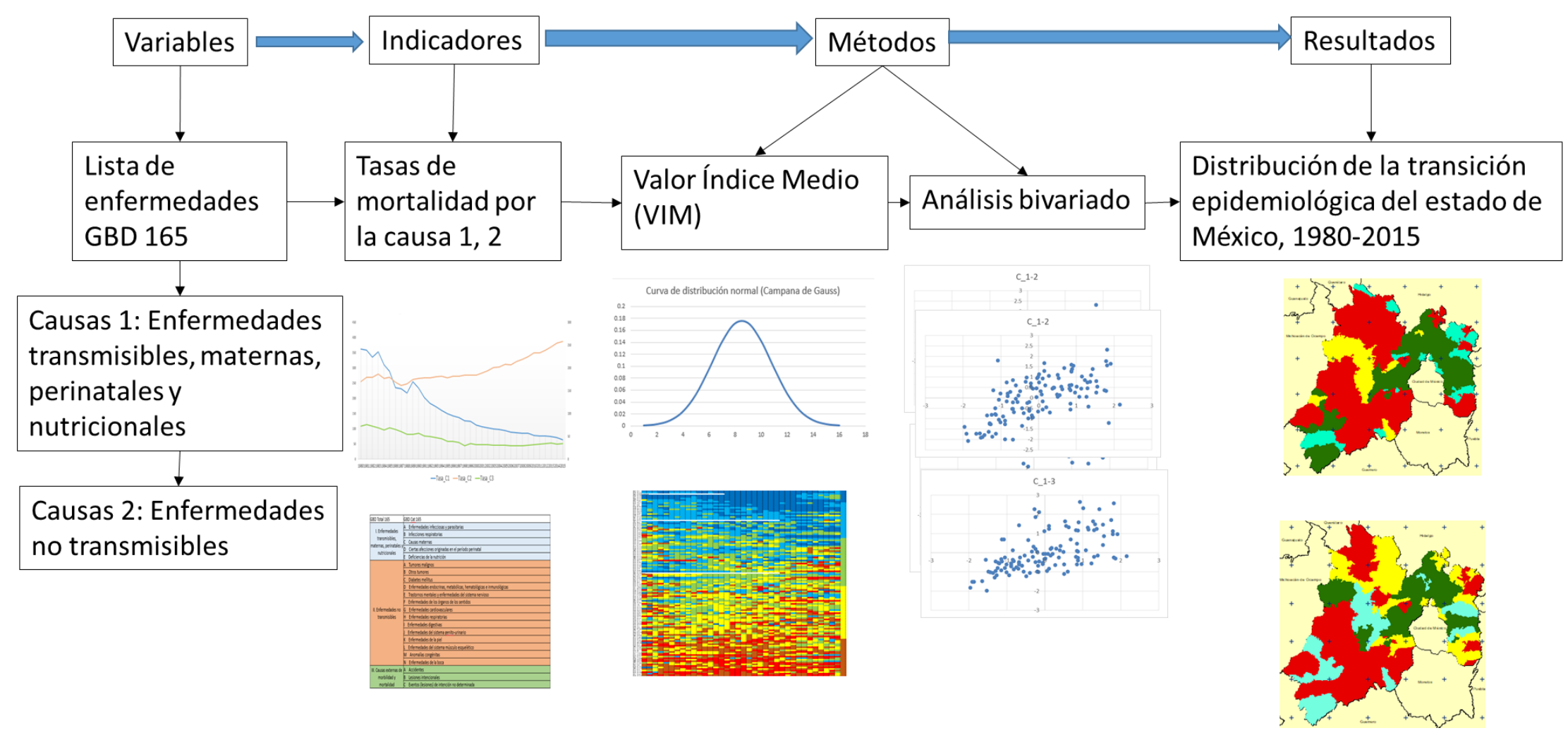




\section{Universo de estudio}

Esta investigación se realizó en el Estado de México, que se encuentra ubicado en el centro de la

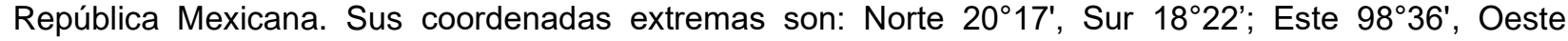
$100^{\circ} 37^{\prime}$. Sus estados colindantes son: al Norte los estados de Querétaro de Arteaga e Hidalgo; al Este, Tlaxcala, Morelos, Puebla y la Ciudad de México; al Sur, Guerrero; y al Oeste, Michoacán de Ocampo. El Estado ocupa una extensión territorial aproximada de 22,357 km² (INEGI, 2020).

El Estado de México presenta una variedad de características físico geográficas, demográficas, sociales, económicas y culturales, es uno de los estados con mayor población en México y presenta contrastes entre los ámbitos urbanos y rurales; la temporalidad de la investigación comprende el periodo 1980-2015, que son tres décadas y media en donde se reflejan los cambios en las causas de muerte, en donde se van desplazando las enfermedades transmisibles y las enfermedades no transmisibles predominan en el territorio y en algunos casos encontrando retrocesos.

\section{Tipo de estudio}

Esta investigación es de tipo descriptiva, cuantitativa y longitudinal. A partir de este periodo de análisis se utilizan las variables sobre defunciones hospitalarias registradas, las causas de enfermedades procedentes de la lista GBD 165, y la población total por año.

\section{Fuentes de información}

Para esta investigación se utilizan los datos de fuentes oficiales siguientes:

La población total del Estado de México (a nivel de municipio) se obtuvo a partir de los Censos de Población y Vivienda realizados en los años 1980, 1990, 2000 y 2010, y a partir de las encuestas intercensales llevadas a cabo en 2005 y 2015 del Instituto Nacional de Estadística y Geografía (INEGI).

Para los datos sobre defunciones hospitalarias se eligieron como criterios el municipio de residencia y fecha de registro, a partir de los cubos dinámicos del Sistema Nacional de Información en Salud (SINAIS).

Con respecto a las causas de enfermedades para la generación de la transición epidemiológica, se retomaron los datos de la lista de enfermedades GBD 165, de 1980-2015.

Con la información recabada se implementó un matriz de datos en el programa Microsoft Excel (2016), en la que se calcularon las tasas de mortalidad y se llevó a cabo el método del valor índice medio. La cartografía se elaboró con el software QGIS (2020).

\section{Variables e indicadores}

Las variables que se retomaron para el estudio de la transición epidemiológica para el Estado de México son:

Número de defunciones por causas de la lista GBD 165, para los años 1980-2015.

Población total de 1980-2015.

A partir de estas variables se calculó la variable de Tasa de Mortalidad por Causa y se generaron los modelos gráficos de la transición epidemiológica.

\section{Procesos y etapas}

El elemento principal para medir la mortalidad es la ocurrencia de las defunciones, que a su vez se clasificaron según su causa, pudiendo ser natural, por enfermedad, por traumatismo o por lesión que conduzca a la muerte. Para esta investigación, se generaron las tasas de mortalidad para las tres causas de muerte correspondientes a la lista de enfermedades GBD 165: enfermedades transmisibles, enfermedades no transmisibles y causas externas de mortalidad y morbilidad.

El cálculo de las tasas se realizó con la siguiente fórmula: 
$\mathrm{TMC}=\frac{D(C L A x)}{P T(C L A x)} * 1000$

Donde:

TMC: Tasa de Mortalidad por Causas

D (CLAx): defunciones debidas a una determinada causa, ocurrida en un lugar y en un año determinado.

PT (CLAx): población total en el mismo lugar y año.

\section{MÉTODOS}

Una vez generadas las tasas de mortalidad por causa, se procedió a elaborar la distribución territorial de la transición epidemiológica.

Para ello, se empleó el método del valor índice medio propuesto por García de León (1989). Para la aplicación de este método, inicialmente se realizó una normalización de la variable de estudio (TMC), con el propósito de evitar extensas diferencias que suelen encontrarse en las variables que hacen referencia a diversos aspectos expresados en unidades distintas (Johnston, 1984 en García de León, 1989). Para esta normalización, inicialmente debe obtenerse la media aritmética y la desviación típica de los datos, con las fórmulas siguientes (Haber, 1973 en García de León, 1989):

Media aritmética de la variable $\mathrm{i}=$

$$
\frac{\sum_{1}^{N} x_{i}}{N}=\frac{x_{1}+x_{2}+x_{3} \ldots+x_{n}}{N}
$$

Desviación típica de la variable i =

$$
\sigma=\sqrt{\frac{\sum_{i}^{N}\left(X_{i}-\bar{X}\right)^{2}}{N}}
$$

Una vez calculadas la media aritmética y de la desviación típica, para las tasas de las causas uno, dos y de mortalidad, se procedió a su normalización empleando la siguiente fórmula:

$$
Z i=\frac{X i-\bar{X} i}{\sigma}
$$

Donde:

i es el número de la variable

$\mathrm{Xi}$ es el valor de cada caso para la variable $\mathrm{i}$

$X i$ es el valor de la media aritmética para la variable i

$\sigma$ es la desviación típica de la variable i

$Z$ es el valor normalizado resultante de la variable i en cada caso 
Evolución territorial de la transición epidemiológica en el estado de México, 1980-2015
Jesús Emilio Hernández Bernal Marcela Virginia Santana Juárez

Noel Bonfilio Pineda Jaimes Jesús Gastón Gutiérrez Cedillo Diana Gómez Barroso

El resultado obtenido de la normalización de las tasas de mortalidad por causas, que se clasificó en seis rangos a partir de la distribución normal o campana de Gauss. Esta distribución es un modelo para un grupo de datos continuo, los cuales se reparten en valores bajos, medios y altos (tabla 1). Posteriormente, según esta clasificación se crea un gráfico de forma acampanada y simétrica, con respecto a un determinado parámetro (Figura 4).

Figura 4 - Curva de distribución normal (campana de Gauss). Fuente: Elaboración propia con base en García de León (1989).

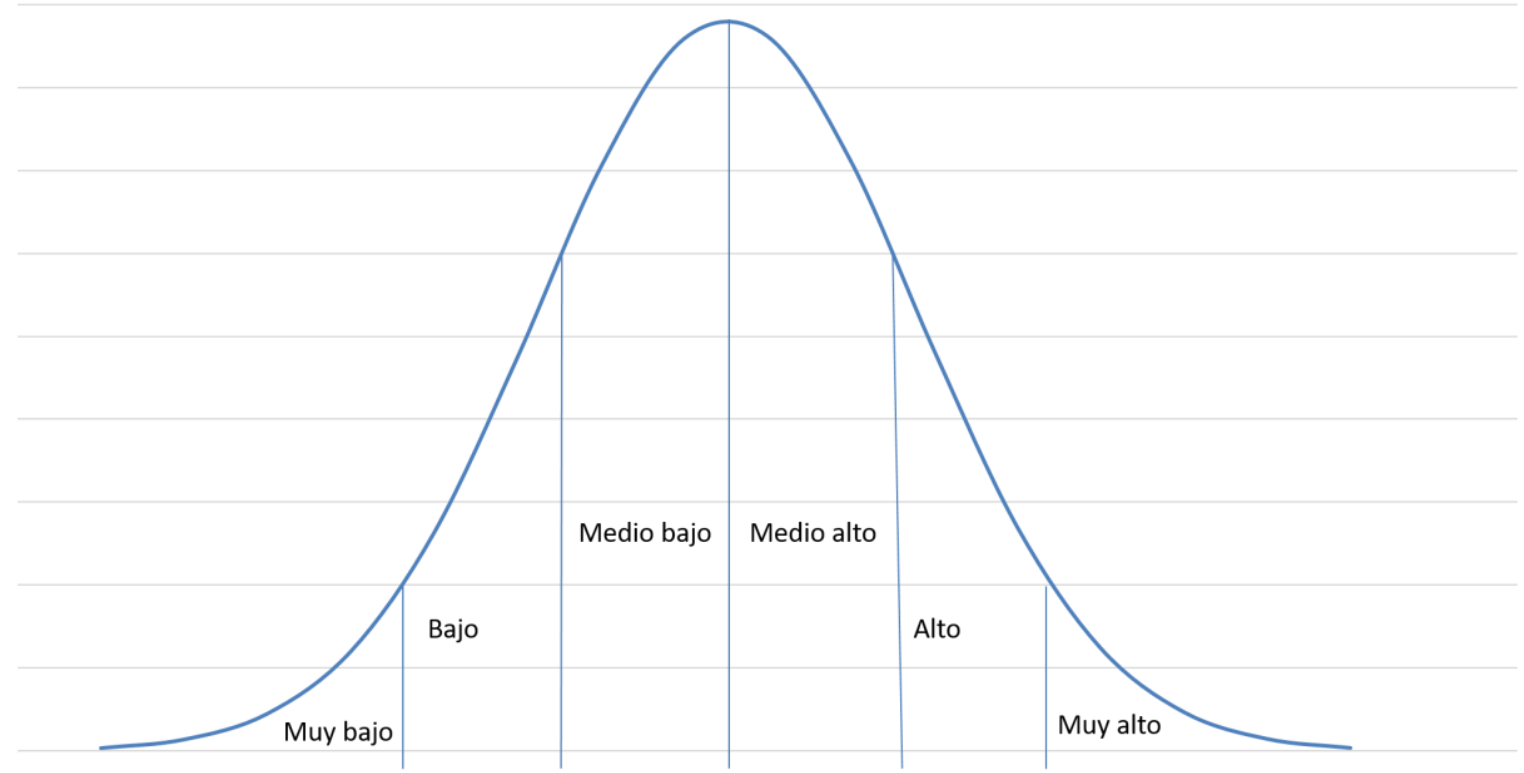

Tabla 1 - Valores de la curva de distribución normal. Fuente: Elaboración propia con base en García de León (1989).

\begin{tabular}{lcc}
\hline \multicolumn{1}{c}{ Simbología } & Desviación estándar & Rango \\
\hline Muy alta & Mayor a 1 & 6 \\
Alta & de 0.5 a 1 & 5 \\
Media-alta & de 0 a 0.5 & 4 \\
Media-baja & de - 0.5 a 0. & 3 \\
Baja & de - 1 a -0.5 & 2 \\
Muy baja & Menor a -1 & 1 \\
\hline
\end{tabular}


El paso final para el análisis según el método del valor índice medio, consistió en el cálculo del promedio de las tasas de mortalidad (TMC para la causa 1 y TMC para la causa 2) para los años 1980 a 2015, con base en los rangos para cada tasa. Si el rango obtenido estaba entre 5 y 6; el promedio sería muy cercano a 6 , si el rango estaba entre 1 y 2 , el promedio resultante sería muy cercano a 2 , y los rangos intermedios deberían de asemejarse a un promedio entre 3 y 4 (García de León, 1989). Como se mencionó, se clasificaron las diferentes causas de muerte en dos grupos (causa 1 y causa 2), de manera que, para la clasificación por periodos, se empleó solo el VIM para cada grupo, quedando únicamente el VIM de la causa 1 y el VIM de la causa 2 para el periodo de 1980-2015.

\section{Método de Análisis bivariado}

Este método permite examinar si existe relación o asociación entre dos variables, en este caso el VIM de la causa 1 y el VIM para la causa 2. Para la obtención de esta asociación, se normalizó el resultado del VIM de las tasas de mortalidad calculadas anteriormente (TMC para la causa 1, y TMC para la causa 2), y se clasificaron los resultados de la normalización. Se asignó una etiqueta de "1" a los valores por encima de la media, y una etiqueta de "0" a los valores que están por debajo de la media, con estos valores estandarizados del VIM se realizó el gráfico de dispersión, donde se compara la distribución de la mortalidad del grupo 1 con respecto al grupo 2

Para la elaboración del mapa temático de la transición demográfica que se presentó en el periodo de estudio, con base en la relación existente entre la tasa mortalidad por causas, se utilizó una clasificación de 4 rangos:

- Alta tasa de la causa 1 y alta tasa de la causa 2 (Transición epidemiológica regresiva).

- Alta tasa de la causa 1 y baja tasa de la causa 2 (Segunda etapa de transición).

- Baja tasa de la causa 1 y alta tasa de la causa 2 (Tercera etapa de transición).

- Baja tasa de la causa 1 y baja tasa de la causa 2 (Transición epidemiológica óptima).

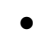

Figura 5 - Gráfico de dispersión de la distribución de las tasas de mortalidad de la causa 1 y la causa 2 .

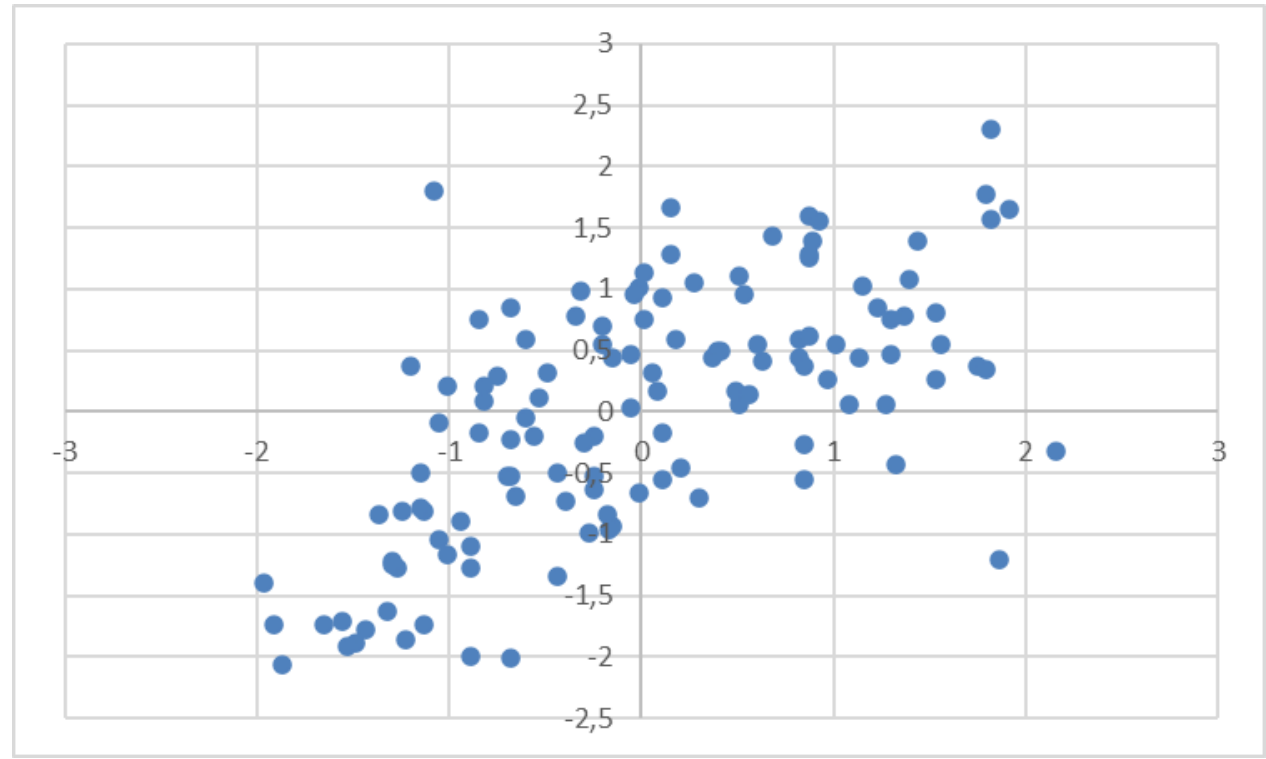

Fuente: Elaboración propia con base en el VIM de la tasa de mortalidad de la causa 1 y 2 (1980-2015). 


\section{RESULTADOS}

En el Estado de México, el comportamiento histórico de la tasa de mortalidad del grupo de la lista de enfermedades GBD 165 se comportó de la manera siguiente:

En el caso de las Enfermedades transmisibles, maternas, perinatales y nutricionales (causa 1), en el año 1980 se registró una tasa de 241 defunciones por cada 100000 habitantes, para el año de 1990 la tasa descendió a 156 defunciones, en 2000 la tasa fue de 75 defunciones, en el año 2010 la tasa registrada fue de 52 defunciones, y para el año 2015 la tasa de mortalidad fue de 42 defunciones por cada 100000 habitantes.

Para las Enfermedades no transmisibles (causa 2), la tasa de mortalidad fue de 256 defunciones por cada 100000 habitantes para el año 1980 García de León (1989)., en 1990 se registraron de 265defunciones, en el año 2000 fue de 277, el año 2010 registró 349 defunciones, y el año 2015 registró una tasa de 387 defunciones por cada 100000 habitantes (tabla 2).

Tabla 2 - Tasa de mortalidad general por 100000 habitantes para el Estado de México, para los años 1980, 1990, 2000, 2010 y 2015, según el tipo de causa.

\begin{tabular}{lccccc}
\hline Tipo de causa & Año 1980 & Año_1990 & Año_2000 & Año_2010 & Año_2015 \\
\hline $\begin{array}{l}\text { I. Enfermedades } \\
\text { transmisibles, } \\
\text { maternas, } \\
\text { perinatales y } \\
\text { nutricionales }\end{array}$ & 241 & 156 & 75 & 52 & 42 \\
$\begin{array}{l}\text { II. Enfermedades } \\
\text { no transmisibles }\end{array}$ & 256 & 265 & 277 & 349 & 387 \\
\hline $\begin{array}{l}\text { III. Causas } \\
\text { externas de } \\
\text { morbilidad y } \\
\text { mortalidad }\end{array}$ & 108 & 84 & & & \\
\hline
\end{tabular}

Fuente: Elaboración propia con base en SINAIS (2020) e INEGI (2020).

La distribución grafica sobre el comportamiento de la transición epidemiológica en el Estado de México (figura 6) incluye las Enfermedades transmisibles, maternas, perinatales y nutricionales (causa 1), Enfermedades no transmisibles (causa 2) y las Causas externas de morbilidad y mortalidad (causa 3). Encontramos un comportamiento a la baja para la causa 1 a lo largo del tiempo, la causa 2 tiende a aumentar su tasa, mientras que la causa 3 desde el inicio ha tenido un comportamiento en descenso, aunque al final del periodo se registró un incremento, que superó el decremento de la causa 1. 
Figura 6 - Transición epidemiológica en el Estado de México para el período 1980 2015, para cada una de las tres causas consideradas.

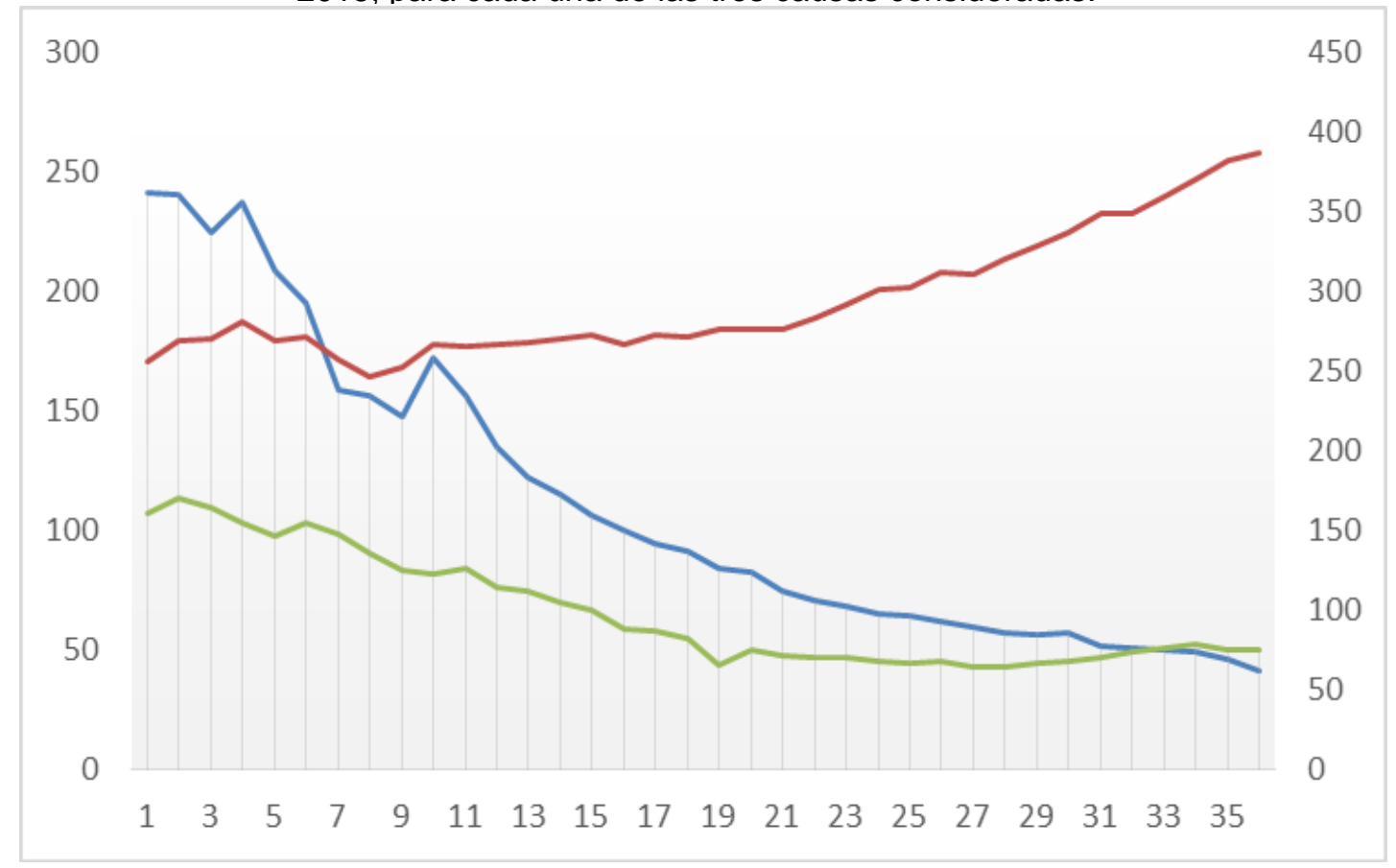

Fuente: elaboración propia.

La transición epidemiológica para el estado de México en el periodo de 1980-2015, presenta un comportamiento en el territorio poco aleatorio, debido a que las etapas de la segunda y tercera transición (zonas con alta tasa de mortalidad de la causa 1 y baja tasa de mortalidad de la causa 2; y zonas con baja tasa de mortalidad de la causa 1 y alta tasa de mortalidad de la causa 2 , respectivamente) se distribuyen territorialmente en la zona centro del estado (causa 1) y en la parte noreste y suroeste del estado (causa 2). La transición epidemiológica regresiva o reemergente (tasa de mortalidad alta de la causa 1 y alta tasa de mortalidad de la causa 2) se distribuye territorialmente en las zonas norte, centrosur y al poniente del estado; la etapa de transición óptima (baja tasa de mortalidad de la causa 1 y una baja tasa de mortalidad por la causa 2) se encuentra ubicada territorialmente en zonas las colindantes a la Ciudad de México, y algunas otras al suroeste del estado (Figura 7). 
Evolución territorial de la transición epidemiológica en el estado de México, 1980-2015
Jesús Emilio Hernández Bernal Marcela Virginia Santana Juárez

Noel Bonfilio Pineda Jaimes Jesús Gastón Gutiérrez Cedillo Diana Gómez Barroso

Figura 7 - Distribución de la transición epidemiológica en el Estado de México para el período 1980 2015, considerando las diferentes etapas de la transición.

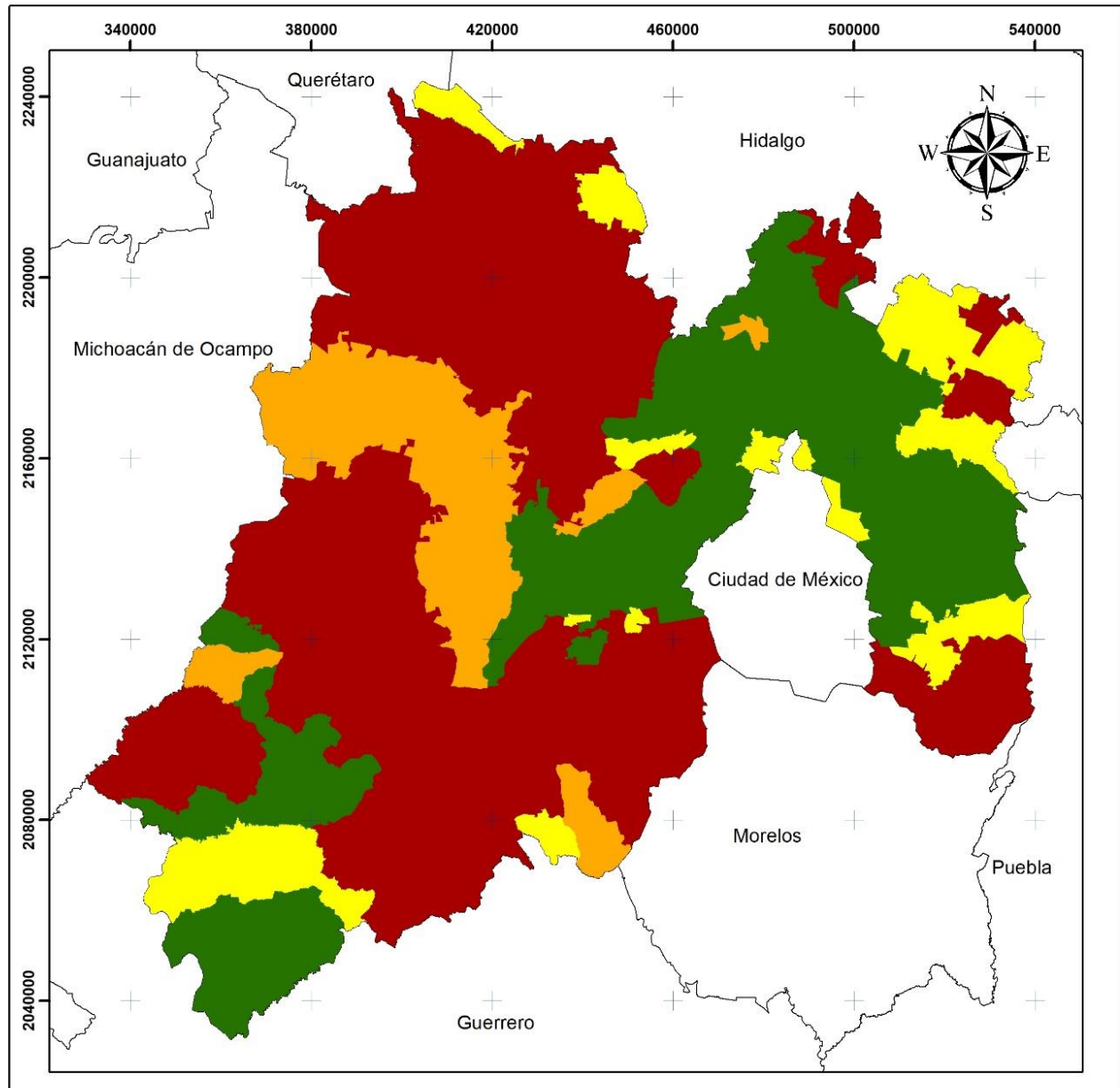

Universidad Autónoma del Estado de México

Distribución de la teoría clásica de la transición epidemiológica del Estado de México, 1980-2015 Simbología:

Segunda etapa de transición
Tercera etapa de transición
Transición óptima
Transición regresiva

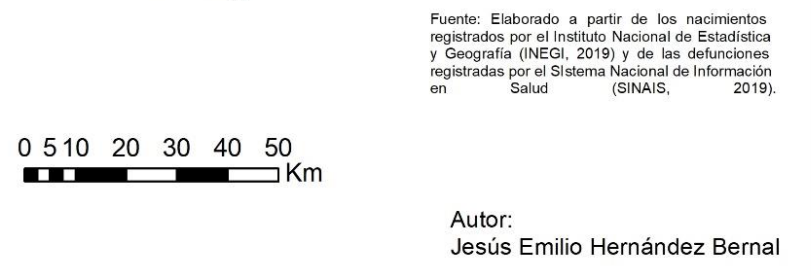

Fuente: elaboración propia.

\section{CONSIDERACIONES FINALES}

Los resultados concluyentes sobre la distribución gráfica y territorial de la transición epidemiológica para el Estado de México en los años 1980-2015, proyectan que el comportamiento gráfico de la transición epidemiológica a través de los años es diferenciado. 
Se concluye que la distribución territorial de la transición epidemiológica para el estado de México en el período 1980-2015 es diferencial en el interior del Estado de México. Se presentan dos etapas dominantes en el territorio: una transición regresiva en la zona norte, centro y suroeste; y una transición óptima en las zonas aledañas a la Ciudad de México. Las áreas que se localizan en la segunda y tercera etapa de transición se distribuyen de manera aleatoria en el Estado de México.

Conocer las diversas etapas de transición epidemiológica en las que se encuentra el Estado de México aporta elementos para la generación de futuras políticas y estrategias en materia de salud, de acuerdo con las características específicas resultantes, con la finalidad de prevenir futuras amenazas de salud y evitar la propagación de estas.

Para elaborar un análisis e identificar el porqué de la distribución en el territorio de la transición epidemiológica, se sugiere considerar variables geográficas, sociales y económicas, además de analizar el impacto de los programas sociales y de salud del Estado de México. Esto será un factor determinante para conocer si en todo el Estado de México se cuenta con las condiciones necesarias para enfrentar los retos en materia de salud dentro del territorio.

Los resultados de esta investigación sobre la distribución grafica de la transición epidemiológica respecto a los estudios previamente consultados tienen cierta similitud, esto se debe a que en las áreas de estudio en donde se realizaron pertenecen a Latinoamérica, estos estudios comprenden períodos mínimo de 10 años en donde se aprecian los cambios en las causas de mortalidad; estas investigaciones generan gráficos por causas tomando en cuenta las que más presencia tienen. En esta investigación además de elaborar el grafico de la transición epidemiológica por la clasificación de enfermedades transmisibles y no trasmisibles, se realizó una representación de la distribución territorial de la transición epidemiológica.

La distribución gráfica y territorial de la transición epidemiológica para el Estado de México durante el período 1980-2015, es general y presenta un acercamiento difuso a la realidad ya que se retomó como base el modelo clásico propuesto por Omran, resultado de esto se reveló que se requiere anexar variables socioeconómicas, demográficas y realizar cortes transversales en determinados años de estudio para identificar el porqué de ese cambio y analizar cuáles son los condicionantes socioeconómicos que influyen en ese cambio, además de analizar el impacto que tendrá a futuro.

\section{BIBLIOGRAFÍA}

Eliecer Andrade, J. (2011). La Transición epidemiológica. Compilación para uso académico. http://bit.ly/356zvBC

Frenk, J., Frejka, T., Bobadilla, J. L., Stern, C., Lozano, R., Sepúlveda, J. y José, M. (1991). La transición epidemiológica en América Latina. Boletín de la Oficina Sanitaria Panamericana (OSP), 111 (6), 485-496.

García de León, A. (1989). La metodología del valor índice medio. Investigaciones geográficas, 19, 69-87. Instituto Nacional de Estadística y Geografía (INEGI). (2020). https://doi.org/10.14350/rig.58975

Kuri-Morales, P.A. (2011). La transición en salud y su impacto en la demanda de servicios. Gaceta Médica de México, 147, 451-454.

Microsoft (2016). Excel (versión 2016) [software de computador].

QGIS (2020). QGIS Sistema de Información Geográfica (versión 3.16.0) [software de computador]. Proyecto de Fundación Geoespacial de Código Abierto. Http://qgis.org

Sistema Nacional de Información en Salud (SINAIS). (2020).

Vera Bolaños, M. (2000). Revisión crítica a la teoría de la transición epidemiológica. Papeles de Población, 6 (25), 179-206. 